TRANSACTIONS OF THE

TRANSACTIONS OF THE
AMERICAN MATHEMATICAL SOCIETY
Volume 243, September 1978

\title{
VECTOR VALUED EIGENFUNCTIONS OF ERGODIC TRANSFORMATIONS
}

BY

\author{
E. FLYTZANIS
}

\begin{abstract}
We study the solutions $X, T$, of the eigenoperator equation

$$
X(h(\cdot))=T X(\cdot) \text { a.e., }
$$

where $h$ is a measurable transformation in a $\sigma$-finite measure space $(S, \Sigma, m)$, $T$ is a bounded linear operator in a separable Hilbert space $H$ and $X$ : $S \rightarrow H$ is Borel measurable. We solve the equation for some classes of measure preserving transformations. For the general case we obtain necessary conditions concerning the eigenoperators, in terms of operators induced by $h$ in the scalar function spaces over the measure space. Finally we investigate integrability properties of the eigenfunctions.
\end{abstract}

Introduction. A measure space $(S, \Sigma, m)$ will denote a separable, $\sigma$-finite measure space. It is called a probability space if $m(S)=1$. A transformation $h: S \rightarrow S$ denotes a measurable invertible nonsingular transformation. It is a measure preserving transformation (m.p.t.) if $m(h(A))=m\left(h^{-1}(A)\right)=m(A)$ for all $A$ in $\Sigma$. $H$ denotes a separable complex Hilbert space, an operator $T$ : $H \rightarrow H$ denotes an invertible bounded linear operator, and $X: S \rightarrow H$ a Borel measurable function. We say that $X, T$ is a solution of the eigenoperator equation for $h$ if

$$
X(h(\cdot))=T X(\cdot) \text { a. e. }
$$

Considered as a generalization of the notion of eigenvalues, eigenoperators present interest for their use in the study of $H$-valued sequences of random variables and also as possible invariants of transformations, in particular measure preserving transformations.

The eigenoperator equation for m.p.t. has been solved completely for the cases where $H$ is finite dimensional or $T$ is unitary, [1], [2]. In a sense these cases are not very interesting because the eigenoperator equation reduces to eigenvalue equations.

In $\S 1$ of this work we find necessary conditions for the eigenoperators $\{T\}$ of a transformation $h$ in terms of a class of Hilbert space operators $\{V\}$

Received by the editors July 7, 1976.

AMS (MOS) subject classifications (1970). Primary 28A65; Secondary 47A10.

Key words and phrases. Eigenvalue, measure preserving transformation, eigenoperator, vector valued eigenfunction, measures in Hilbert space, weighted shift operators.

- American Mathematical Society 1978 
induced by $h$ in $L_{2}(m)$ where $V f(\cdot)=f(h(\cdot))$. These conditions are shown to be also sufficient for some classes of m.p.t. and solutions $X, T$ for which $X$ is square integrable. In $\$ 2$ we study integrability properties of eigenfunctions for ergodic transformations.

In this work we make use of some basic notions from the theory of m.p.t. and the unitary operators induced by them [3], [4], also from the theory of Hilbert-Schmidt operators [5]. Concerning conventions we note the following: Many statements referring to the measure space hold modulo sets of measure zero, but usually we will not mention this explicitly. If $X, T$ is a solution of the eigenoperator equation we denote by $H(X)$ the subspace of $H$ spanned by the essential range of $X$ and then $H(X)$ is invariant under both $T$ and $T^{-1}$. Also $X(\cdot) \in H(X)$ a.e. and clearly we can study only the restriction of $T$ to $H(X)$.

1. Eigenoperators. $h,(S, \Sigma, m)$ are as in the introduction. We say that $h$ is $m$-bounded if $m\left(h^{-1}(A)\right)<c m(A)$ and $m(h(A))<c m(A)$ for some $c>1$ and all $A$ in $\Sigma$. In this case we say also that $m$ is $h$-bounded. We note that given $h$ and $m$ we can always find a probability measure $m^{\prime}$ equivalent to $m$ $\left(m^{\prime} \sim m\right)$ and $h$-bounded. Thus, if $m$ is already a probability measure we can define $m^{\prime}$ by

$$
m^{\prime}(A)=\sum m\left(h^{i}(A)\right) / 3 \cdot 2^{|i|}
$$

for every $A$ in $\Sigma$, otherwise we find first an equivalent probability measure, which we can do because the space is $\sigma$-finite, and then proceed as above.

LEMma 1. If $m^{\prime}, m^{\prime \prime}$ are two equivalent $\sigma$-finite measures and $m^{\prime \prime}$ is $h$ bounded, then $m^{\prime}$ is also $h$-bounded iff the positive (a.e.) functions

$$
w\left(h^{-1}(\cdot)\right) / w(\cdot) \text { and } w(h(\cdot)) / w(\cdot)
$$

are (ess.) bounded, where $w(\cdot)=d m^{\prime} / d m^{\prime \prime}$ is the Lebesgue derivative of $m^{\prime}$ with respect to $m^{\prime \prime}$.

Proof. Omitted.

LEMMA $2 . h$ is a transformation in a $\sigma$-finite measure space $(S, \Sigma, m)$ and $X, T$ is a solution of the eigenoperator equation. Then we can find a probability measure $m^{\prime} \sim m$ such that:

a. $m^{\prime}$ is $h$-bounded.

b. $X$ is square integrable with respect to $m^{\prime}$, i.e. $\int\|X\|^{2} d m^{\prime}<\infty$.

PROOF. First we find as indicated above a probability measure $m^{\prime \prime} \sim m$ that is also $h$-bounded. Then we define a probability measure $m^{\prime} \sim m^{\prime \prime}$ by its Lebesgue derivative $c w(\cdot)=d m^{\prime} / d m^{\prime \prime}$ where:

$$
w(s)=1 \text { if } s \in S^{\prime}=\{s:\|X(s)\|<1\}
$$


and

$$
w(s)=1 /\|X(s)\|^{2} \quad \text { if } s \notin S^{\prime} .
$$

We have $w(\cdot)<1$ and $c$ is chosen so that $m^{\prime}(S)=1$. Concerning the ratio $w\left(h^{-1}(\cdot)\right) / w(\cdot)$ we have: if $s \in S^{\prime}$ then $w(s)=1$ and

$$
w\left(h^{-1}(s)\right) / w(s)=w\left(h^{-1}(s)\right)<1 ;
$$

if $s \notin S^{\prime}$ and $h^{-1}(s) \notin S^{\prime}$, then

$$
w\left(h^{-1}(s)\right) / w(s)=\|X(s)\|^{2} /\left\|X\left(h^{-1}(s)\right)\right\|^{2} \leqslant\|T\|^{2}
$$

by the eigenoperator equation; finally if $s \notin S^{\prime}$ and $h^{-1}(s) \in S^{\prime}$ then $\left\|X\left(h^{-1}(s)\right)\right\|<1$ and

$$
\begin{aligned}
w\left(h^{-1}(s)\right) / w(s) & =1 / w(s)=\|X(s)\|^{2}=\left\|X\left(h\left(h^{-1}(s)\right)\right)\right\|^{2} \\
& <\|T\|^{2}\left\|X\left(h^{-1}(s)\right)\right\|^{2} \leqslant\|T\|^{2}
\end{aligned}
$$

by the eigenoperator equation. Therefore

$$
w\left(h^{-1}(\cdot)\right) / w(\cdot) \leqslant \max \left\{1,\|T\|^{2}\right\} .
$$

In the same way we show

$$
w(h(\cdot)) / w(\cdot)<\max \left\{1,\left\|T^{-1}\right\|^{2}\right\}
$$

and by Lemma 1 it follows that $m^{\prime}$ is $h$-bounded. Concerning b we have:

$$
\int\|X\|^{2} d m^{\prime}=\int_{S^{\prime}}\|X\|^{2} d m^{\prime}+\int_{\bar{S}^{\prime}}\|X\|^{2} d m^{\prime}=m^{\prime}\left(S^{\prime}\right)+m^{\prime \prime}\left(\bar{S}^{\prime}\right)<\infty \text {. Q.E.D. }
$$

Given a transformation $h$ in a $\sigma$-finite measure space $(S, \Sigma, m)$ we define a class of Hilbert space operators as follows: if $m$ is $h$-bounded it induces in $L_{2}(m)$ an invertible bounded linear operator $V_{h}$, where $V_{h} f(\cdot)=f(h(\cdot))$; in general we denote by $L_{h}$ the class $\left\{V_{h, m^{\prime}}\right\}$ of Hilbert space invertible bounded linear operators, where $m^{\prime} \sim m$ is a $h$-bounded probability measure and $V_{h, m^{\prime}} f(\cdot)=f(h(\cdot))$.

Proposition 1. $h$ is a transformation in a $\sigma$-finite measure space $(S, \Sigma, m)$. $X, T$ is a solution to the eigenoperator equation for $h$ with $H(X)=H$. Then

(i) There exists an operator $V \in L_{h}$ and a Hilbert-Schmidt operator $K$ whose range is dense in $H$ such that

$$
K V^{*}=T K
$$

where $V$ is the adjoint of $V$.

(ii) If $m$ is $h$-bounded and $X$ is square integrable with respect to $m$ then in (2) we can replace $V$ by $V_{h}$, i.e.

$$
K V_{h}^{*}=T K
$$


Proof. (i) First we find a probability measure $m^{\prime}$ as in Lemma 1. Next we define $K: L_{2}\left(m^{\prime}\right) \rightarrow H$ by the strong integral

$$
K f=\int f(\cdot) X(\cdot) d m^{\prime}, \quad f \in L_{2}\left(m^{\prime}\right) .
$$

A similar operator appears also in the study of probability measure in Hilbert spaces [5]. $K$ is well defined as a bounded linear operator, in fact

$$
\|K\|^{2}<\int\|X(\cdot)\|^{2} d m^{\prime}<\infty
$$

by Lemma 2. To show that $K$ is Hilbert-Schmidt we consider an orthonormal basis $\left\{f_{i}\right\}$ in $L_{2}\left(m^{\prime}\right)$. Let also $\left\{v_{j}\right\}$ be an orthonormal basis in $H$. Then

$$
\begin{aligned}
\sum_{i}\left\|K f_{i}\right\|^{2} & =\sum_{i} \sum_{j}\left|\left\langle K f_{i}, v_{j}\right\rangle\right|^{2}=\sum_{i} \sum_{j}\left|\int f_{i}(\cdot)\left\langle X(\cdot), v_{j}\right\rangle d m^{\prime}\right|^{2} \\
& =\sum_{j} \int\left|\left\langle X(\cdot), v_{j}\right\rangle\right|^{2} d m^{\prime}=\int\|X(\cdot)\|^{2} d m^{\prime}<\infty .
\end{aligned}
$$

It follows that $K$ is Hilbert-Schmidt. To show that the range of $K$ is dense in $H$ we note that if $v$ is a vector in $H$ then $\langle X(\cdot), v\rangle \in L_{2}\left(m^{\prime}\right)$ and not the zero a.e. function because of $H(X)=H$. Therefore for some $f_{i} \in\left\{f_{i}\right\}$ we have

$$
\left\langle K f_{i}, v\right\rangle=\int f(\cdot)\langle X(\cdot), v\rangle d m^{\prime} \neq 0 .
$$

Finally we note that

$$
\begin{aligned}
T K f & =\int f(\cdot) T X(\cdot) d m^{\prime}=\int f(\cdot) X(h(\cdot)) d m^{\prime} \\
& =\int f\left(h^{-1}(\cdot)\right) X(\cdot) d\left(m^{\prime} \circ h^{-1}\right)=K V^{*} f,
\end{aligned}
$$

because $V^{*} f=f\left(h^{-1}(\cdot)\right) \cdot\left(d\left(m^{\prime} \circ h^{-1}\right) / d m^{\prime}\right)$.

(ii) We can replace $m^{\prime}$ by $m$ in the proof for (i). Q.E.D.

Unfortunately not much is known about the operators in $L_{h}$, while $V_{h}$ is well understood especially if $m$ is $h$-invariant in which case $V_{h}$ is unitary. For the rest of this section we consider m.p.t. on $\sigma$-finite measure spaces. Also we consider only solution to the eigenoperator equation having square integrable eigenfunctions. From Proposition 1 (ii) we can derive a complete characterization of eigenoperators in this case. An operator $\Lambda: H \rightarrow H$ is called an $S$-operator [5] if it is selfadjoint, positive semidefinite and its eigenvalues have finite sum.

THEOREM 1. $T$ is an eigenoperator of a m.p.t. in a $\sigma$-finite measure space having square integrable eigenfunction with $H(X)=H$ iff there exists an $S$-operator $\Lambda: H \rightarrow H$ injective and with dense range such that $T \Lambda T^{*}=\Lambda$. 
Proof. $(\Leftarrow)$. In $H$ we define the Gaussian probability measure having covariance operator $\Lambda$ and zero mean. Then $T$ preserves this measure [5] and the identity function on $H$ is the required eigenfunction. $(\Rightarrow)$ From equation $K V_{h}^{*}=T K$ in Proposition 1 (ii) it follows that the orthogonal complement of the kernel of $K$ is invariant under both $V_{h}$ and $V_{h}^{-1}=V_{h}^{*}$. Restricting the equation to this subspace we have that $K$ is injective and has dense range. Taking adjoints we find $V_{h} K^{*}=K^{*} T$ where $K^{*}$ is also injective and has dense range. Multiplying the two equations we obtain $T \Lambda T^{*}=\Lambda$ where $\Lambda=K K^{*}$ has all the required properties. Q.E.D.

REMARK. It follows from the proof above that we can obtain all eigenoperators of m.p.t. with square integrable eigenfunctions, by considering only probability spaces.

The next result, obtained in [2] by a different approach follows now directly from Theorem 1 .

CoROllary 1. If $H$ is finite dimensional or if $T$ is unitary then $T$ is an eigenoperator of a m.p.t., having an eigenfunction as in Theorem 1 , iff $H$ is spanned by eigenvectors of $T$ having eigenvalues of norm 1 . Then it follows from Proposition 1 that they are also eigenvalues of $h$.

Next we solve the eigenoperator equation for some classes of m.p.t. For convenience we consider only ergodic m.p.t. $L_{2}^{\prime}(m)$ denotes the subspace of $L_{2}(m)$ consisting of functions with zero mean, i.e. $\int f(\cdot) d m=0$. By the spectrum of $h$ we mean the spectrum of the restriction of $V_{h}$ to $L_{2}^{\prime}(m)$. We note that Proposition 1 (ii) implies that the images under $K$ of the orbits of $V_{h}^{*}$ are orbits of $T$.

THEOREM 2. $h$ is an ergodic m.p.t. having complete point spectrum. Then $T$ is an eigenoperator of h having square integrable eigenfunction with $H(X)=H$, iff $H$ is spanned by eigenvectors of $T$ having distinct eigenvalues that are also eigenvalues of $h$.

Proof. $(\Rightarrow)$ It follows from Proposition 1 (ii). $(\Leftarrow)\left\{v_{i}: i=1,2, \ldots\right\},\left\{c_{i}\right.$ : $i=1,2, \ldots\}$ are the eigenvectors and their corresponding eigenvalues, as assumed in the theorem. If $\left\{f_{i}: i=1,2, \ldots\right\}$ are the corresponding eigenfunctions of $h$ then

$$
X(\cdot)=\sum_{i} f_{i}(\cdot) v_{i} / 2^{|i|}
$$

has all the required properties. Q.E.D.

Next we consider ergodic transformations with continuous spectrum. In this case we assume for convenience that the eigenfunction has zero mean, i.e. $\int X(\cdot) d m=0$, and then we can take the restriction of the equation $K V_{h}^{*}=$ $T K$ to the subspace $L_{2}^{\prime}(m)$. We assume also $H(X)=H$. We can derive 
various orbit structure properties of $T$ by using known properties of $V_{h}^{*}=$ $V_{h}^{-1}$, [4]. The collection $\{v\} \subset H$ consisting of the nonzero elements of $K L_{2}^{\prime}(m)$ is dense in $H$. Thus for every $v$ in a dense subset $\{v\}$ of $H$ we have:

a. The orbits $\left\{T^{i} v: i=1,2, \ldots\right\},\left\{T^{i} v: i=-1,-2, \ldots\right\}$ are infinite dimensional, precompact and have the zero vector as an accumulation point.

b. They have the ergodic property,

$$
\left(\sum_{1}^{n} T^{i} v / n\right) \rightarrow \text { zero vector strongly, } \quad \text { as } n \rightarrow \pm \infty .
$$

c. If $h$ has absolutely continuous spectrum then $T^{i} v \rightarrow$ zero vector strongly, as $i \rightarrow \pm \infty$.

In deriving the conditions above we only use the fact that $K$ is compact. Perhaps the work in [6] can be used to derive some stronger conditions using also the properties of $K$ as a Hilbert-Schmidt operator.

In the special case where $h$ has $\sigma$-Lebesgue spectrum, which arises often in applications, we can in fact derive necessary and sufficient conditions.

THEOREM 3. $h$ is an ergodic m.p.t. having $\sigma$-Lebesgue spectrum. $T$ is an eigenoperator of $h$ having a square integrable eigenfunction with zero mean and $H(X)=H$, iff there exists a countable collection $\left\{v_{i}: i=1,2, \ldots\right\} \subset H$ such that:

a. $\left\{T^{i} v_{j}: i=1,2, \ldots, j=0, \pm 1, \pm 2, \ldots\right\}$ spans $H$, and

b. $\Sigma_{i} \Sigma_{j}\left\|T^{i} v_{j}\right\|^{2}<\infty$.

Proof. If $h$ has $\sigma$-Lebesgue spectrum we can find a countably infinite collection $\left\{f_{k}: k=1,2, \ldots\right\} \subset L_{2}^{\prime}(m)$ such that

$$
\left\{f_{k}\left(h^{j}(\cdot)\right): k=1,2, \ldots, j=0, \pm 1, \ldots\right\}
$$

is an orthonormal basis for $L_{2}^{\prime}(m)$. Then: $(\Rightarrow)$ follows from Proposition 1 (ii) using the defining property of Hilbert-Schmidt operators. $(\Leftarrow)$ The function

$$
X(\cdot)=\sum_{i} \sum_{j} f_{i}\left(h^{-j}(\cdot)\right) T^{j} v_{i}
$$

satisfies all the requirements. Q.E.D.

If in the operators of Theorem 3 we impose also the condition that $\left\{T^{i} v_{j}\right\}$ is an orthogonal collection then we obtain basically weighted shift operators which are eigenoperators of every m.p.t. although it is not clear what $H(X)$ would be in each case.

2. Eigenfunctions. As it appears that the integrability properties of eigenfunctions are important we examine this question. We assume now that $h$ is an ergodic transformation in a probability space $(S, \Sigma, m)$. If $X, T$ is a solution and $\|T\| \leqslant 1$ or $\left\|T^{-1}\right\| \leqslant 1$ then $X$ must have constant norm (a.e.) and in 
particular it is $p$-integrable for every $p>0$ [1], as it is also if $T$ or $T^{-1}$ are totally bounded, i.e.

$$
\left\|T^{n}\right\|, \quad n=0,1,2, \ldots, \text { or }\left\|T^{-n}\right\|, \quad n=0,1,2, \ldots,
$$

are uniformly bounded. In order to state a result for the general case we introduce first some notation. The spectral radius of an operator $T: H \rightarrow H$ is defined by $r(T)=\lim \sup \left\|T^{n}\right\|^{1 / n}$. Also we define the spectral radius $r(h)$ for an ergodic transformation in a probability space as follows. For every $\boldsymbol{A}$ with $m(A)>0$ we define the sets

$$
A_{n}=\bigcup_{-n}^{n} h^{i}(A), \quad n=0,1,2, \ldots, \quad \triangle A_{n}=A_{n}-A_{n-1}, \quad n=1,2, \ldots
$$

The sets $\triangle A_{n}$ are disjoint and $m\left(U \triangle A_{n}\right)=1$. The radius of convergence of the power series $\Sigma c^{n} m\left(\triangle A_{n}\right)$ is given by

$$
r(A, h)=1 / \lim \sup \left(m\left(\triangle A_{n}\right)\right)^{1 / n} .
$$

We define $r(h)=\inf \{r(A, h): A \in \Sigma, m(A)>0\}$. Clearly $r(h)>1$.

PROPOSITION 2. $h$ is an ergodic transformation in a probability space and $r(h)$ its spectral radius.

(i) If $X, T$ is a solution of the eigenoperator equation and

$$
r(T), r\left(T^{-1}\right)<(r(h))^{1 / p}
$$

then $X$ is p-integrable. Also if $r(A, h)>r(h)$ for all $A$ then the statement holds also with equality.

(ii) For every $\varepsilon>0$ and $p>0$ there exists a solution $X, T$ such that

$$
\|T\|,\left\|T^{-1}\right\|<(r(h))^{1 / p}+\varepsilon
$$

and $X$ is not p-integrable, provided $H$ is infinite dimensional. Also if $r(A, h)=$ $r(h)=1$ for some $A$ then we can find the same solution for all $p$.

Proof. (i) We set $f(\cdot)=\|X(\cdot)\|^{p}$ and $c_{n}=\max \left\{\left\|T^{n}\right\|^{p},\left\|T^{-n}\right\|^{p}\right\}$. From the eigenoperator equation we have $f\left(h^{n}(\cdot)\right), f\left(h^{-n}(\cdot)\right)<c_{n} f(\cdot)$. Also for some $\alpha>0$ we have $m(A=\{s: f(s)<a\})>0$. We define the function $g(\cdot)$ by $g(s)=a c_{n}$ for $s \in \Delta A_{n}, n=0,1, \ldots$. Then $\int g(\cdot) d m=a \Sigma c_{n} m\left(\Delta A_{n}\right)<$ $\infty$ because

$$
\begin{aligned}
\lim \sup \left(c_{n} m\left(\triangle A_{n}\right)\right)^{1 / n} & <\lim \sup \left(c_{n}\right)^{1 / n} \lim \sup \left(m\left(\triangle A_{n}\right)\right)^{1 / n} \\
& <(r(T))^{p} / r(h)<1 .
\end{aligned}
$$

(If $r(A, h)>r(h)$ we can replace the last $<$ by $<$.) Since $f(\cdot) \leqslant g(\cdot)$ by the definition of $g(\cdot)$ it follows that $f(\cdot)$ is integrable and hence $X(\cdot)$ is $p$-integrable.

(ii) If $r(h)=\infty$ there is nothing to prove. Otherwise we choose $c=$ $(r(h))^{1 / p}+\varepsilon / 2$ and a set $A \in \Sigma$ so that $r(A, h)<r(h)+p \varepsilon / 2$. We define 
$g(\cdot)$ by $g(s)=c_{n}$ for $s \in \triangle A_{n}$. Then

$$
\int g^{p}(\cdot) d m=\sum\left(c^{p}\right)^{n} m\left(\triangle A_{n}\right)=\infty \text { because } c^{p}>r(A, h) .
$$

(If $r(A, h)=r(h)=1$ we would take this $A$.) Next we choose

$$
a=(r(h))^{1 / p}+\varepsilon=c+\varepsilon / 2
$$

and if $\left\{v_{n}: n=0, \pm 1, \ldots\right\}$ is an orthonormal set in $H$ we define $T: H \rightarrow H$ by $T v_{n}=v_{n+1} / a$ for $n \geqslant 0$ and $T v_{n}=a v_{n+1}$ for $n<0$. The $H$-valued function

is well defined because

$$
X(\cdot)=\sum g\left(h^{-n}(\cdot)\right) v_{n} / a^{|n|}
$$

$$
\|X(\cdot)\| \leqslant \sum g\left(h^{-n}(\cdot)\right) a^{-|n|} \leqslant g(\cdot) \sum c^{|n|} / a^{|n|}<\infty \text { a.e. }
$$

$X$ is not $p$-integrable because $\|X(\cdot)\|^{p} \geqslant g^{p}(\cdot)$. Finally we note that $X, T$ is a solution and

$$
\|T\|=\left\|T^{-1}\right\|=a=(r(h))^{1 / p}+\varepsilon . \quad \text { Q.E.D. }
$$

It follows that $h$ has all its eigenfunctions integrable with respect to $m$ iff $r(h)=\infty$. However no such transformation or even transformations with $r(h) \neq 1$ are known. One can also compute $r(h)$ using intersections of sets and then if $A$ is such that

$$
\left\{h^{i}(A): i=0, \pm 1, \ldots\right\}
$$

are independent, where $h$ is $m . p . t$. , then

$$
r(A, h)=1 / m(\bar{A})^{2},
$$

and if there exists such sets with measure arbitrarily close to zero we get $r(h)=1$. Thus if $h$ is the two-sided shift on the infinite product space of the unit interval then $r(h)=1$, and we can apply Proposition 2 (ii).

We add that Proposition 2 holds also for ergodic transformations on infinite $\sigma$-finite measure spaces if in this case we define $r(h)=1$.

\section{REFERENCES}

1. A. Beck, Eigenoperators of ergodic transformations, Trans. Amer. Math. Soc. 94 (1960), 118-129. MR 27 \#2606.

2. E. Flytzanis, Linear dynamical systems, Proc. Amer. Math. Soc. 55 (1976), 367-370.

3. P. R. Halmos, Lectures on ergodic theory, Chelsea, New York, 1960. MR 20 \#3958; 22 \#2677.

4. K. Jacobs, Lecture notes on ergodic theory, 1962/63, Part I, Mat. Inst. Aarhus Univ., Aarhus, 1963. MR 28 \#3138; 28, p. 1247.

5. H. H. Kuo, Gaussian measures in Banach spaces, Springer-Verlag, Berlin and New York, 1975.

6. U. Krengel, Weakly wandering vectors and weakly independent partitions, Trans. Amer. Math. Soc. 164 (1972), 199-226. MR 44 \# 7353.

School of Technology, Aristotelian University of Thessaloniki, Thessaloniki, Greece 